\title{
Modelling short-term Solar Spectral Irradiance (SSI) using coronal electron density and temperature profiles based on solar magnetic field observations
}

\author{
J. M. Rodríguez Gómez ${ }^{1}$, L. E. Antunes Vieira ${ }^{1}$, A. Dal Lago ${ }^{1}$, \\ J. Palacios ${ }^{2}$, L. A. Balmaceda ${ }^{3}$ and T. Stekel ${ }^{1}$ \\ ${ }^{1}$ National Institute for Space Research (INPE), \\ Avenida dos Astronautas-12227-010, São José dos Campos-SP, Brazil \\ email: jenny.gomez@inpe.br \\ ${ }^{2}$ Universidad de Alcalá. Pza. San Diego, s/n 28801 - Alcalá de Henares (Madrid) España. \\ ${ }^{3}$ Instituto de Ciencias Astronómicas de la Tierra y el Espacio, ICATE-CONICET, Avda. de \\ España Sur 1512, J5402DSP, San Juan, Argentina.
}

\begin{abstract}
Some key physical processes that impact the evolution of Earth's atmosphere on timescale from days to millennia, such as the EUV emissions, are determined by the solar magnetic field. However, observations of the solar spectral irradiance are restricted to the last few solar cycles and are subject to large uncertainties. We present a physics-based model to reconstruct short-term solar spectral irradiance (SSI) variability. The coronal magnetic field is estimated to employ the Potential Field Source Surface extrapolation (PFSS) based on observational synoptic charts and magnetic flux transport model. The emission is estimated to employ the CHIANTI atomic database 8.0. The performance of the model is compared to the emission observed by TIMED/SORCE.
\end{abstract}

Keywords. Sun: magnetic fields, photosphere, corona.

\section{Introduction}

Solar Spectral Irradiance, which is the energy flux from the Sun at specific wavelengths, influences the dynamics of the space weather and the Earth's atmosphere. Extreme Ultraviolet irradiance (EUV) originates in the corona and chromosphere. It is as ionizing radiation, it is absorbed by Earth's upper atmosphere, ionizing atoms and splitting molecules. This process creates the Earth's ionosphere. The EUV band plays an important function in the thermodynamics and the chemistry of the Earth's upper atmosphere (Solanki et al. 2013, Meier, R.R. 1991).

The Earth's climate is influenced by solar variability. This variability represents about $8 \%$ of the total radiative forcing. The Solar Spectral Irradiance (SSI) variations influence the ozone photochemistry and radiative heating. Models of SSI variations are important in climate studies. Reliable SSI series of realistic simulations are necessary to describe the atmospheric chemistry (Ermolli et al. 2013).

Mechanisms have been proposed to explain the spectrally-resolved solar electromagnetic radiation (SSI), in general, their are caused by the magnetic features on the solar surface. The relationship between the irradiance and solar magnetic field is known. The magnetic field is strengthened and maintained in the solar interior by the solar dynamo action. It is observed as a variety of phenomena in the solar atmosphere (Mackay \& Yeates 2012, Solanki et al. 2006, Hargreaves 1995, Low, B.C. 1996). 
The magnetic solar dynamo can generate large-scale and small-scale magnetic field. The poloidal and toroidal magnetic field components are related to solar atmospheric phenomena. Shearing of poloidal field by differential rotation produces toroidal field, when the toroidal field is strong enough, buoyant loops rise at the surface. The sunspots are formed from these loops and flux emerges. It is spread in latitude and longitude along the activity belt. Meridional flow carries surface magnetic flux, some of this flux is then transported (Dikpati \& Gilman 2009). Variations of photospheric magnetic flux and the evolution of magnetic features have been proposed to explain the origin and variation of solar irradiance (Ermolli et al. 2013, Solanki et al. 2013, Vieira et al. 2011, Krivova et al. 2007).

The variability of the SSI is driven by different physical processes. The solar cycle (11- year) is guided by the global magnetic field evolution. Emergence and decay of the magnetic regions and modulations of the solar rotation happens. Differences in irradiance measurements at various wavelengths are due to the radiation emitted at different heights in the solar atmosphere. The temperature in magnetic features drops more slowly with height in the solar photosphere and increase much more rapidly with height in the chromosphere. Thus the radiation emitted at higher levels in the solar atmosphere shown larger changes (Ermolli et al. 2013).

Observations of the solar irradiance are limited to the last few solar cycles and are showing some uncertainties related to instrumental degradation and interrupted observations. The need to alleviate these observational problems has encouraged the development of SSI models. In the present work we have reconstructed variability of short-term Solar Spectral Irradiance (SSI) in the EUV band at $17.1 \mathrm{~nm}$. For this purpose, we use a flux transport model, the structure of coronal magnetic field obtained using Potential Field Source Surface (PFSS) and an emission model.

\section{Data}

We have used synoptic magnetogram data from the MDI instrument aboard of the Solar and Heliospheric Observatory (SOHO) (Scherrer et al. 1995) and the HMI instrument aboard of the Solar Dynamics Observatory (SDO) (Scherrer et al. 2012). The magnetic synoptic map is a proxy of the entire surface distribution of the photospheric magnetic field. The synoptic map of the solar magnetic field is assembled from individual magnetograms observed over the course of a solar rotation $\sim 27$ days.

\section{Approach}

In order to reconstruct the solar spectral irradiance (SSI), the basic assumption is the evolution of the emission which is based on the configuration of the magnetic field imprinted on the solar surface. We used solar surface magnetic field from the surface flux model of Schrijver (2001). The surface flux model was updated each six hours. This model considers flux emergence, differential rotation, meridional and dispersal flows over the solar photosphere. The Potential Field Source Surface (PFSS) was used to extrapolate the line-of-sight surface magnetic field from the photosphere $r=1 R_{\odot}$ up to the corona $r=2.5 R_{\odot}$.

We consider the magnitude of the magnetic field $B$ through the solar corona. The electron density $(N)$ and temperature $(T)$ profiles are functions of the magnitude of the magnetic field, for more information we refer the reader to the proceeding of the IAU symposium 328: Rodríguez et al. 2016 (Maresias, Brazil). We used the emission model to build from CHIANTI atomic database 8.0 (Del Zanna et al. 2000). Coronal 


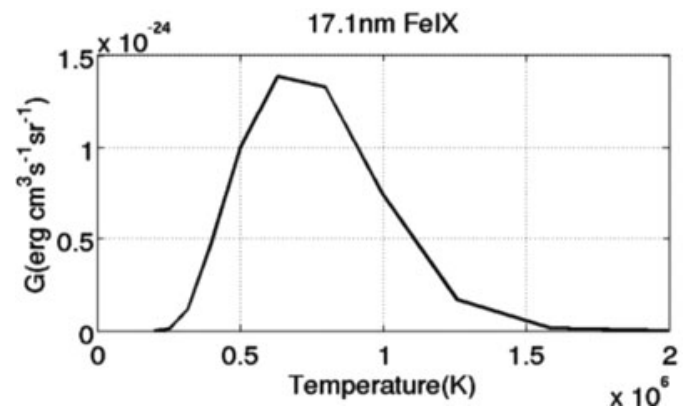

Figure 1. Contribution function $G(\lambda, T)$ from the Chianti atomic database 8.0 at $17.1 \mathrm{~nm}$ FeIX line.

abundances from Meyer (1985) and ionization equilibrium from Mazzotta et al. (1998) were used to compute the contribution function $G(\lambda, T)$ (Fig. 1). It was considered the central wavelength Fe IX line at $17.1 \mathrm{~nm}$. The contribution function $(G(\lambda, T))$ contains all of the relevant atomic physical parameters such as ionization equilibrium and coronal abundances. The emission line depends on the atomic transitions and the conditions of the solar atmosphere, the intensity in each voxel is described by:

$$
I_{o}=\iint R(\lambda) G(\lambda, T) d \lambda N^{2} d s
$$

where $G(\lambda, T)\left[\mathrm{erg} \mathrm{cm} \mathrm{cm}^{-1} \mathrm{sr}^{-1}\right]$ is the contribution function from the CHIANTI atomic database 8.0, $d \lambda[\mathrm{nm}]$ is spectral resolution, $N\left[\mathrm{~cm}^{-3}\right]$ is the electron density and $d s[\mathrm{~cm}]$ is the differential distance along the line-of-sight and $R(\lambda)$ is the instrumental response.

\section{Preliminary results}

We study the evolution of the Solar Spectral Irradiance (SSI) during the two last solar cycles. For this purpose, we use magnetic field from synoptic charts of MDI/SOHO and HMI/SDO instruments. We obtained the coronal density and temperature profiles. These profiles were used to obtain the emission (Equation (3.1)) in a specific wavelength.

The reconstruction of short-term Solar Spectral Irradiance (SSI) at $17.1 \mathrm{~nm}$ from our approach is shown in Fig. 2. This result is in agreement with observational data from time series of TIMED/SORCE.

\section{Discussion}

The SSI at $17.1 \mathrm{~nm}$ is related to the variations and evolution of photospheric magnetic through the solar cycle. Our approach describes the relationship between the EUV emission and the evolution of the magnetic field due to active regions and their decay. The dynamics of the magnetic field is provided by the flux transport model (Schrijver 2001). This model describes the flux emergence, differential rotation, meridional flow, dispersal and disappear flux. The trend of SSI in 17.1nm follows the solar cycle, with the irradiance being higher during cycle phases with higher activity and small values of irradiance that coincide with the passage of sunspots across the solar disk.

The description of the emission in each line is related to the atomic transitions, coronal abundances and the conditions of the solar atmosphere provided by CHIANTI atomic 


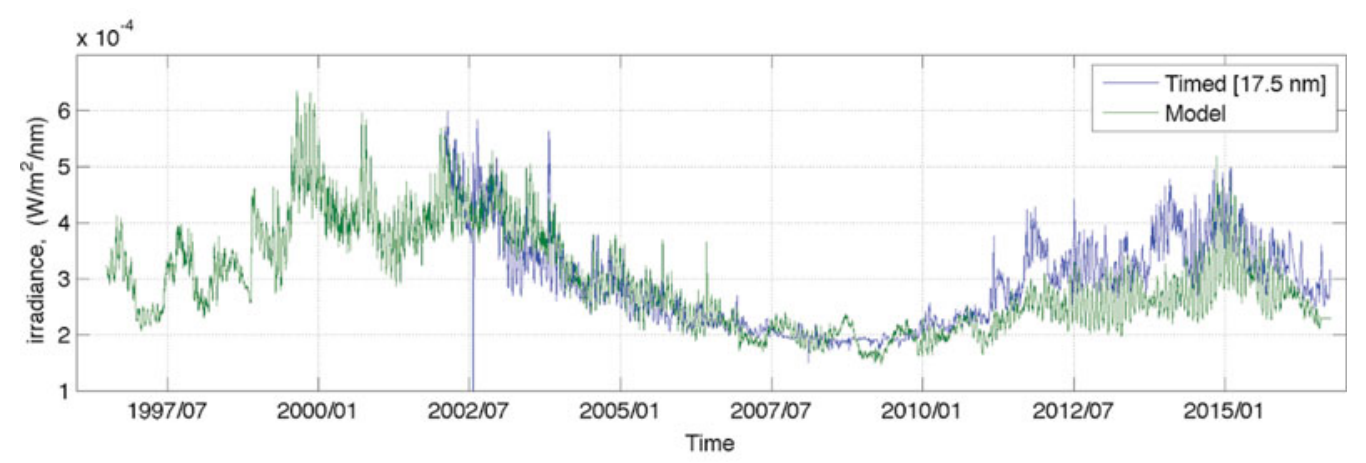

Figure 2. The Solar Spectral Irradiance (SSI) from the solar cycle 23 to 24 . Our approach is shown from 1996 to 2015; and the observational data (TIMED/SORCE) from 2002 to 2015.

database 8.0. It constitutes a better approach to reconstruct the solar coronal dynamics and the Solar Spectral Irradiance necessary to the climate models. This study can contribute to analyse the long-term emission and properties in the solar corona.

\section{Acknowledgements}

We would like to thank SOHO/MDI, SDO/HMI Data Science and Teams and the Chianti atomic database 8.0. This work is partially supported by CNPq/Brazil under the grant agreement no. 140779/2015-9 and no. 304209/2014-7. JP acknowledges UAH travel grant for visiting INPE and MINECO project no. AYA2013-47735-P.

\section{References}

Dikpati, M. \& Gilman, P. 2009, SSRv, 144, 67

Ermolli, I., Matthes, K., Dudok de Wit, T., Krivova, N. A., Tourpali, K., Weber, M., Unruh, Y. C., Gray, L., Langematz, U., Pilewskie, P., Rozanov, E., Schmutz, W., Shapiro, A., Solanki, S. K., \& Woods, T. N. 2013, ACP, 13, 8

Hargreaves, J. K. 1995, Camb. Atmos. Space Sci. Ser., 5

Krivova, N. A., Balmaceda, L., \& Solanki, S. K. 2007, A\&\&A, 467, 1

Low, B. C. 1996, Solar Phys., 167, 1

Mackay, D. H. \& Yeates, A. R. 2012, LRSP, 9, 6

Mazzotta, P., Mazzitelli, G., Colafrancesco, S., \& Vittorio, N. 1998, A\&\A, 133, 403

Meier, R. R. 1991, Space Science Reviews, 58, 1

Meyer, J. 1985, ApJS, 57, 173

Pesnell, W. D., Thompson, B. J., \& Chamberlin, P. C. 2012, Solar Phys., 275, 3

Scherrer, P. H., Schou, J., Bush, R. I., Kosovichev, A. G., Bogart, R. S., Hoeksema, J. T., Liu, Y., Duvall, T. L., Zhao, J., Title, A. M., Schrijver, C. J., Tarbell, T. D., \& Tomczyk, S. 2012, Solar Phys., 275, 207

Scherrer, P. H., Bogart, R. S., Bush, R. I., Hoeksema, J. T., Kosovichev, A. G., Schou, J., Rosenberg, W., Springer, L., Tarbell, T. D., Title, A., Wolfson, C. J., \& Zayer, I., MDI Engineering Team 1995, Solar Phys., 162, 129

Schrijver,C. \& De Rosa, M. 2003, Solar Phys., 212, 165

Schrijver,C. 2001, ApJ, 547, 475

Solanki, S. K., Inhester, B., \& Schussler, M. 2006, Solar Phys., 69, 3

Solanki, S. K., Krivova, N. A., \& Haigh, J. D. 2013, ARA $\& A$, 51, 1

Tripathi, D., Mason, H. E., Young, P. R., \& Del Zanna, G. 2008, A\& $A$, 481, 1

Vieira, L. E. A., Solanki, S. K., Krikova, N. A., \& Usoskin,I. 2008, A\&A, 531, A6 